\title{
Dimensionamento amostral para avaliação de altura e diâmetro de mudas de nogueira-pecã
}

\author{
Sample size for evaluating the height and diameter of seedlings pecan
}

\author{
Alberto Cargnelutti Filho ${ }^{\text {I }}$ Tales Poletto $^{\text {II }}$ Marlove Fátima Brião Muniz $^{\text {III }}$ Carine Baggiotto $^{\text {IV }}$ \\ Igor Polettov Diniz Fronza ${ }^{\mathrm{VI}}$
}

\begin{abstract}
- NOTA -
RESUMO

O objetivo deste trabalho foi determinar o tamanho de amostra (número de plantas) necessário para a estimação da média de altura de planta, do diâmetro do coleto e da relação altura de planta/diâmetro do coleto de mudas de nogueira-pecã (Carya illinoinensis) e verificar a variabilidade do tamanho de amostra entre tratamentos de superação de dormência de sementes. Foram avaliados 12 tratamentos de superação de dormência de sementes e, aos 98 dias após a semeadura, foram

seed dormancy and at 98 days after sowing it was measured the plant height $(H)$ and collar diameter $(D)$ and calculated the relation plant height/collar diameter (HD). Measures of central tendency and variability were calculated, the normality and homogeneity of variances were verified and sample size was calculated. The sample size to evaluate $H, D$ e $H D$ is dependent on treatments of overcoming seed dormancy. Sixty-three plants enough to predict the average of $H, D$ and $H D$ seedlings pecan, with an estimation error equal to $10 \%$ of estimated average, with a degree confidence of $95 \%$.
\end{abstract} mensurados os caracteres altura de planta $(H)$ e diâmetro do coleto $(D)$, e calculada a relação altura de planta/diâmetro do coleto $(H D)$. Foram calculadas medidas de tendência central e de variabilidade, verificadas a normalidade e a homogeneidade de variâncias e calculado o tamanho de amostra. $O$ tamanho de amostra para avaliar $H, D$ e $H D$ de mudas de nogueira-pecã é dependente do tratamento para superação de dormência de sementes. Sessenta e três plantas são suficientes para a estimação da média de $H$, do D e da HD de mudas de nogueira-pecã, para erro de estimação de $10 \%$ da média estimada, com grau de confiança de $95 \%$.

Palavras-chave: Carya illinoinensis, planejamento experimental, amostragem.

\section{ABSTRACT}

The aim of this research was to determine the sample size (number of plants) necessary to estimate the average plant height, collar diameter and relation plant height/collar diameter of seedlings pecan (Carya illinoinensis) and to verify the variability of the sample size among treatments of overcoming seed dormancy. It was evaluated 12 treatments of overcoming
Key words: Carya illinoinensis, experimental planning, sampling.

Para o estabelecimento de plantios de nogueira-pecã (Carya illinoinensis [Wangenh] K. Koch), é importante ter mudas de qualidade adequada. Na produção de mudas de nogueira-pecã, em viveiros florestais, têm sido utilizados métodos de superação de dormência das sementes. Em experimentos conduzidos em viveiro florestal, é importante avaliar, com precisão desejada, caracteres como a altura de planta, o diâmetro do coleto e a relação altura de planta/diâmetro do coleto, durante o crescimento das plantas. Para isso, é importante dimensionar o número de plantas que devem ser avaliadas para possibilitar inferências precisas sobre os tratamentos em avaliação.

IDepartamento de Fitotecnia, Centro de Ciências Rurais (CCR), Universidade Federal de Santa Maria (UFSM), 97105-900, Santa Maria, RS, Brasil. E-mail: alberto.cargnelutti.filho@gmail.com. Autor para correspondência.

IICurso de Engenharia Florestal, UFSM, Santa Maria, RS, Brasil.

IIIDepartamento de Defesa Fitossanitária, CCR, UFSM, Santa Maria, RS, Brasil.

${ }^{\text {IV }}$ Curso de Engenharia Sanitária e Ambiental, UFSM, Santa Maria, RS, Brasil.

${ }^{\mathrm{v} U n i v e r s i d a d e ~ F e d e r a l ~ d o ~ P a m p a ~(U N I P A M P A), ~ S a ̃ o ~ G a b r i e l, ~ R S, ~ B r a s i l . ~}$

${ }^{\mathrm{VI}}$ Departamento de Fruticultura, Colégio Politécnico, UFSM, Santa Maria, RS, Brasil. 
O tamanho de amostra para mudas de Eucalyptus saligna, em viveiro florestal, para diferença mínima significativa de $10 \%$, foi de 23 e 26 mudas por tratamento, respectivamente, para a altura de planta e o diâmetro do coleto (ZANON et al., 1997). Para as variáveis altura e diâmetro do coleto, o tamanho de amostra para mudas de Pinus taeda L., em viveiro florestal, variou em função da idade das mudas (SILVEIRA et al., 2009). Esses autores determinaram que o tamanho de amostra para semiamplitude de $10 \%$ foi de 25 mudas para a altura e de 12 mudas para o diâmetro do coleto. Para avaliar a altura de planta, o diâmetro do coleto e a relação altura de planta/diâmetro do coleto de mudas de Cabralea canjerana (CARGNELUTTI FILHO et al., 2012), concluíram que o tamanho de amostra depende da idade de avaliação e que 18 plantas por unidade experimental (bandeja) são suficientes para a estimação da média desses caracteres, para erro de estimação de $10 \%$ da média.

Nãoforam encontrados na literatura estudos sobre o dimensionamento amostral para a avaliação de mudas de nogueira-pecã. Assim, o objetivo deste trabalho foi determinar o tamanho de amostra (número de plantas) necessário para a estimação da média de altura de planta, do diâmetro do coleto e da relação altura de planta/diâmetro do coleto de mudas de nogueira-pecã e verificar a variabilidade do tamanho de amostra entre tratamentos de superação de dormência de sementes.

Foi conduzido um experimento em ambiente controlado, com temperatura de $25^{\circ} \mathrm{C} \pm 2^{\circ} \mathrm{C}$ e fotoperíodo de 12 horas, com mudas de nogueira-pecã, no Laboratório de Fitopatologia do Departamento de Defesa Fitossanitária da Universidade Federal de Santa Maria, Santa Maria, Rio Grande do Sul (RS). Em maio de 2012, foram coletadas sementes de nogueira-pecã (variedade Barton) de 25 árvores matrizes, em pomares comerciais no município de Anta Gorda, RS. Essas sementes, com massa superior a $10 \mathrm{~g}$, foram lavadas em água corrente para eliminar os restos de polpa e, posteriormente, colocadas em local sombreado, por três semanas, para a secagem. Em seguida, foram separadas 12 amostras de sementes para aplicação dos 12 tratamentos de superação de dormência (T1, T2, ..., T12). Nos tratamentos T1, T5 e T9, as sementes foram mantidas em temperatura ambiente, dentro de sacos de papel, no escuro, e armazenadas no Laboratório de Fitopatologia, durante 30, 60 e 90 dias, respectivamente. Esses tratamentos foram considerados testemunhas, pois não foi aplicado método auxiliar de superação de dormência. Nos tratamentos T2, T6 e T10, as sementes foram mantidas nas mesmas condições anteriores, durante 30, 60 e 90 dias, respectivamente e, após, foi escarificada a parte apical, com lixa para madeira, número 80. Nos tratamentos T3, T7 e T11, as sementes foram estratificadas e acomodadas em camadas alternadas de areia, com $5 \mathrm{~cm}$ de espessura, dispostas em bandejas plásticas providas de orifícios na parte inferior para drenagem do excesso de água. Essas bandejas foram mantidas em câmara fria à temperatura de $4^{\circ} \mathrm{C} \pm 0,5^{\circ} \mathrm{C}$, durante 30,60 e 90 dias, respectivamente, para os tratamentos T3, T7 e T11. Nos tratamentos T4, T8 e T12, as sementes foram escarificadas na parte apical e, posteriormente, estratificadas, conforme descrito anteriormente, e permaneceram nessa condição durante 30, 60 e 90 dias, respectivamente.

Ao término de cada tratamento, foram semeadas 30 sementes, sendo 15 em cada bandeja de plástico de $35 \mathrm{~cm}$ de comprimento, $25 \mathrm{~cm}$ de largura e $10 \mathrm{~cm}$ de altura, contendo substrato comercial. Para evitar possíveis influências do meio, as bandejas foram alternadas de posição semanalmente. Aos 98 dias após a semeadura (DAS), foi mensurada a altura de planta $(\mathrm{H})$, em $\mathrm{cm}$, com régua milimetrada, e o diâmetro do coleto (D), em $\mathrm{mm}$, com paquímetro digital, e calculada a relação altura de planta/ diâmetrodo coleto (HD), em $\mathrm{cm} \mathrm{mm}^{-1}$.

Para cada caractere (H, D e HD) mensurado nas plantas amostradas de cada tratamento, foram calculadas as estatísticas: número de plantas (n), valores mínimo e máximo, média $(\mathrm{m})$, mediana, variância, desvio-padrão (s), erro-padrão e coeficiente de variação. Foi verificada a normalidade, por meio do teste de Kolmogorov-Smirnov, totalizando 36 testes (12 tratamentos $\times 3$ caracteres). A seguir, aplicou-se o teste $\mathrm{F}$ (bilateral), a $5 \%$ de probabilidade, para testar a hipótese de homogeneidade das variâncias entre os tratamentos (12 variâncias) em cada caractere (3 testes). $\mathrm{O}$ valor do teste $\mathrm{F}$ (bilateral) foi calculado pela razão entre os tratamentos com maior e menor variância ( $\mathrm{F}=$ maior variância/menor variância), com $\mathrm{n}-1$ graus de liberdade no numerador e $\mathrm{m}-1$ graus de liberdade no denominador.

Para os caracteres $\mathrm{H}, \mathrm{D}$ e HD, em cada tratamento, a partir do número de plantas 
amostradas (n), da média amostral (m) e do desviopadrão amostral (s), foi calculado o tamanho de amostra $(\eta)$ para as semiamplitudes do intervalo de confiança (erro de estimação), fixadas em $1 \%, 2 \%, \ldots$, $15 \%$ da média amostral $(\mathrm{m})$, ou seja, $0,01 \times \mathrm{m}$ (maior precisão), $0,02 \times \mathrm{m}, \ldots, 0,15 \times \mathrm{m}$ (menor precisão), com grau de confiança $(1-\alpha)$ de $95 \%$, por meio da expressão $\eta=\left[\left(\mathrm{t}_{\alpha / 2} \mathrm{~s}\right) /(\text { erro de estimação) }]^{2}\right.$ (BUSSAB \& MORETTIN, 2011), na qual $\mathrm{t}_{\alpha / 2}$ é o valor crítico da distribuição $\mathrm{t}$ de Student, tal que $\mathrm{P}\left(\mathrm{t}>\mathrm{t}_{\alpha / 2}\right)=\alpha / 2$, com $\alpha=5 \%$ de probabilidade de erro e (n-1) graus de liberdade, e s é o desvio-padrão amostral. As análises estatísticas foram realizadas com os aplicativos GENES (CRUZ, 2013) e Microsoft Office Excel ${ }^{\circledR}$.

Entre os 12 tratamentos de superação de dormência, as médias de altura de planta (H), diâmetro do coleto (D) e relação altura de planta/diâmetro do coleto (HD) oscilaram, respectivamente, entre 15,18 e $23,30 \mathrm{~cm}, 3,01$ e $4,09 \mathrm{~mm}$ e 4,04 e $7,20 \mathrm{~cm} \mathrm{~mm}^{-1}$ (Tabela 1), o que sugere que, para esses três caracteres, há variabilidade entre os tratamentos. Análise de variância, seguida de testes de comparações múltiplas de médias, seriam procedimentos adequados para a identificação do(s) tratamento(s) que proporcionariam mudas com desempenho superior, em relação à $\mathrm{H}$, ao $\mathrm{D}$ e à HD. Portanto, para conclusões definitivas sobre possíveis diferenças de H, D e HD de mudas de nogueirapecã entre os tratamentos de superação de dormência de sementes, estudos mais detalhados devem ser realizados e não foi o foco deste trabalho.

Em relação à $\mathrm{H}$, ao $\mathrm{D}$ e à $\mathrm{HD}$, os valores mínimos e máximos e as medidas de dispersão (variância, desvio-padrão, erro-padrão e coeficiente de variação), em cada tratamento (Tabela 1), refletem a variabilidade existente entre as plantas e, consequentemente, a necessidade de calcular o tamanho de amostra para estimação da média. O teste F (bilateral), aplicado para testar a hipótese de homogeneidade de variâncias entre os tratamentos (12 variâncias), revelou que as variâncias foram heterogêneas para os caracteres $\mathrm{H} \quad(\mathrm{F}=5,14$, valor- $\mathrm{p}=0,0005), \mathrm{D}(\mathrm{F}=3,09$, valor- $\mathrm{p}=0,0196)$ e $\mathrm{HD}$ $(\mathrm{F}=5,66$, valor- $\mathrm{p}=0,0001)$, o que indica que o tamanho de amostra difere entre os tratamentos. Assim, conclui-se que o tamanho de amostra para avaliar $\mathrm{H}$, D e HD de mudas de nogueira-pecã é dependente do tratamento de superação de dormência de sementes. Variabilidade do tamanho de amostra entre idades de avaliação (tratamentos) também foi constatada em mudas de Pinus taeda (SILVEIRA et al., 2009) e de Cabralea canjerana (CARGNELUTTI FILHO et al., 2012).

O coeficiente de variação $(\mathrm{CV})$ médio dos 12 tratamentos foi de $26,10 \%, 25,21 \%$ e $23,22 \%$, respectivamente, para $\mathrm{H}, \mathrm{D}$ e $\mathrm{HD}$, o que sugere que o tamanho de amostra para a estimação da média de $\mathrm{H}$, D e HD, com mesma precisão, decresce nessa ordem. Variabilidade do tamanho de amostra entre caracteres já foi constatada em mudas de Eucalyptus saligna (ZANON et al., 1997), de Pinus taeda (SILVEIRA et al., 2009) e de Cabralea canjerana (CARGNELUTTI FILHO et al., 2012).

De maneira geral, para os 36 casos (12 tratamentos $\times 3$ caracteres), a proximidade da média em relação à mediana e os elevados valores-p (valor-p $\geq 0,207$ ) do teste de KolmogorovSmirnov (Tabela 1), indicam boa aderência dos dados à distribuição normal. Assim, pode-se inferir que os dados são adequados para o estudo do dimensionamento amostral, com base na distribuição t de Student.

O tamanho de amostra, para a estimação da média de H, D e HD, com erro de estimação igual a $1 \%$ da estimativa da média (m) (maior precisão), oscilou entre 987 plantas para HD do T4 e 6.295 para H do T3 (Tabela 2). Portanto, a estimação da média de H, D e HD, nesses 12 tratamentos, com essa precisão, é difícil de ser praticada, pelo elevado número de plantas a serem mensuradas. Opcionalmente, o pesquisador pode, a partir dos tamanhos de amostra, com erros de estimação de $1 \%$ a $15 \%$ (Tabela 2), definir a combinação entre tamanho de amostra e precisão que lhe seja satisfatória. Como exemplo, na prática, para esses 12 tratamentos de superação de dormência de sementes, 63 plantas são suficientes para estimação da média de H, D e HD de mudas de nogueira-pecã, para erro de estimação de $10 \%$ da média estimada, com grau de confiança de $95 \%$.

\section{AGRADECIMENTOS}

Ao Conselho Nacional de Desenvolvimento Científico e Tecnológico ( $\mathrm{CNPq})$, pela concessão de bolsa de Produtividade em Pesquisa para Alberto Cargnelutti Filho e Marlove Fátima Brião Muniz. 
Tabela 1 - Número de plantas (n), valores mínimo e máximo, média, mediana, variância, desvio-padrão, erro-padrão, coeficiente de variação (CV\%) e valor-p do teste de normalidade de Kolmogorov-Smirnovpara três caracteres de mudas de nogueira-pecã (Carya illinoinensis), avaliados em 12 tratamentos (TRAT) de superação de dormência de sementes.

\begin{tabular}{|c|c|c|c|c|c|c|c|c|c|c|}
\hline $\mathrm{TRAT}^{(1)}$ & $\mathrm{n}$ & Mínimo & Máximo & Média & Mediana & Variância & Desvio-padrão & Erro-padrão & $\mathrm{CV}(\%)$ & Valor-p \\
\hline & \multicolumn{10}{|c|}{------------------------------------------------ } \\
\hline $\mathrm{T} 1$ & 21 & 4,60 & 22,50 & 15,18 & 15,60 & 22,84 & 4,78 & 1,04 & 31,49 & 0,989 \\
\hline $\mathrm{T} 2$ & 22 & 8,40 & 23,30 & 17,29 & 17,30 & 12,08 & 3,48 & 0,74 & 20,10 & 0,953 \\
\hline $\mathrm{T} 3$ & 25 & 2,00 & 24,00 & 15,25 & 16,50 & 34,37 & 5,86 & 1,17 & 38,44 & 0,633 \\
\hline $\mathrm{T} 4$ & 27 & 9,80 & 23,00 & 17,56 & 18,80 & 15,89 & 3,99 & 0,77 & 22,70 & 0,659 \\
\hline $\mathrm{T} 5$ & 22 & 10,50 & 23,70 & 17,04 & 17,00 & 13,24 & 3,64 & 0,78 & 21,36 & 0,999 \\
\hline T6 & 19 & 9,10 & 22,30 & 16,72 & 17,40 & 15,34 & 3,92 & 0,90 & 23,43 & 0,988 \\
\hline $\mathrm{T} 7$ & 28 & 10,50 & 34,30 & 19,19 & 18,85 & 21,82 & 4,67 & 0,88 & 24,35 & 0,539 \\
\hline $\mathrm{T} 8$ & 28 & 9,00 & 26,50 & 17,80 & 17,60 & 13,66 & 3,70 & 0,70 & 20,76 & 0,983 \\
\hline T9 & 28 & 14,50 & 36,50 & 23,30 & 23,65 & 38,75 & 6,22 & 1,18 & 26,72 & 0,728 \\
\hline $\mathrm{T} 10$ & 21 & 4,50 & 33,00 & 21,63 & 22,10 & 62,04 & 7,88 & 1,72 & 36,42 & 0,820 \\
\hline $\mathrm{T} 11$ & 26 & 15,00 & 32,00 & 21,56 & 19,70 & 21,40 & 4,63 & 0,91 & 21,46 & 0,207 \\
\hline $\mathrm{T} 12$ & 29 & 13,50 & 35,00 & 21,87 & 21,00 & 32,13 & 5,67 & 1,05 & 25,92 & 0,866 \\
\hline $\mathrm{T} 1$ & 21 & 1,51 & 5,26 & 3,01 & 3,03 & 1,03 & 1,01 & 0,22 & -----------------------------------' & 0,933 \\
\hline $\mathrm{T} 2$ & 22 & 2,18 & 5,18 & 3,44 & 3,38 & 0,71 & 0,84 & 0,18 & 24,45 & 0,822 \\
\hline $\mathrm{T} 3$ & 25 & 1,80 & 6,23 & 3,70 & 3,76 & 0,93 & 0,97 & 0,19 & 26,07 & 0,933 \\
\hline $\mathrm{T} 4$ & 27 & 1,69 & 5,78 & 3,66 & 3,80 & 0,81 & 0,90 & 0,17 & 24,55 & 0,991 \\
\hline $\mathrm{T} 5$ & 22 & 1,49 & 4,20 & 3,12 & 3,20 & 0,54 & 0,73 & 0,16 & 23,44 & 0,559 \\
\hline T6 & 19 & 2,00 & 4,39 & 3,18 & 3,22 & 0,41 & 0,64 & 0,15 & 20,13 & 0,981 \\
\hline $\mathrm{T} 7$ & 28 & 2,31 & 6,24 & 3,92 & 3,81 & 0,67 & 0,82 & 0,15 & 20,82 & 0,704 \\
\hline T8 & 28 & 1,59 & 5,83 & 3,83 & 3,82 & 0,88 & 0,94 & 0,18 & 24,55 & 0,668 \\
\hline T9 & 28 & 1,51 & 5,90 & 3,36 & 3,41 & 0,98 & 0,99 & 0,19 & 29,41 & 0,945 \\
\hline $\mathrm{T} 10$ & 21 & 1,12 & 5,47 & 3,10 & 3,25 & 1,27 & 1,12 & 0,25 & 36,26 & 0,979 \\
\hline $\mathrm{T} 11$ & 26 & 2,42 & 5,92 & 3,97 & 3,87 & 0,79 & 0,89 & 0,17 & 22,36 & 0,690 \\
\hline \multirow[t]{2}{*}{$\mathrm{T} 12$} & 29 & 2,94 & 5,70 & 4,09 & 4,03 & 0,47 & 0,69 & 0,13 & 16,79 & 0,868 \\
\hline & \multicolumn{10}{|c|}{-------------------------------------Relação altura de planta/diâmetro do coleto $\left(\mathrm{cm} \mathrm{mm}^{-1}\right)$--- } \\
\hline $\mathrm{T} 1$ & 21 & 3,05 & 9,38 & 5,28 & 4,83 & 3,14 & 1,77 & 0,39 & 33,56 & 0,476 \\
\hline $\mathrm{T} 2$ & 22 & 3,69 & 8,26 & 5,18 & 4,90 & 1,54 & 1,24 & 0,26 & 23,92 & 0,643 \\
\hline T3 & 25 & 1,11 & 5,94 & 4,04 & 4,27 & 1,77 & 1,33 & 0,27 & 32,94 & 0,707 \\
\hline $\mathrm{T} 4$ & 27 & 3,36 & 6,50 & 4,87 & 4,76 & 0,55 & 0,74 & 0,14 & 15,28 & 0,917 \\
\hline T5 & 22 & 3,41 & 7,72 & 5,63 & 5,42 & 1,47 & 1,21 & 0,26 & 21,51 & 0,936 \\
\hline T6 & 19 & 2,85 & 8,03 & 5,35 & 5,13 & 1,52 & 1,23 & 0,28 & 23,00 & 0,866 \\
\hline $\mathrm{T} 7$ & 28 & 3,16 & 6,79 & 4,97 & 5,00 & 1,00 & 1,00 & 0,19 & 20,10 & 0,983 \\
\hline T8 & 28 & 3,32 & 7,75 & 4,81 & 4,63 & 1,11 & 1,05 & 0,20 & 21,93 & 0,495 \\
\hline T9 & 28 & 4,49 & 10,67 & 7,20 & 7,08 & 2,96 & 1,72 & 0,32 & 23,87 & 0,979 \\
\hline $\mathrm{T} 10$ & 21 & 3,84 & 9,36 & 7,04 & 7,28 & 2,52 & 1,59 & 0,35 & 22,54 & 0,869 \\
\hline $\mathrm{T} 11$ & 26 & 4,14 & 8,33 & 5,55 & 5,21 & 1,35 & 1,16 & 0,23 & 20,96 & 0,610 \\
\hline $\mathrm{T} 12$ & 29 & 3,34 & 7,47 & 5,33 & 5,01 & 1,03 & 1,02 & 0,19 & 19,03 & 0,579 \\
\hline
\end{tabular}

${ }^{(1)} \mathrm{T} 1$, sementes em temperatura ambiente por 30 dias; T2, sementes em temperatura ambiente por 30 dias e escarificadas após esse período; T3, sementes estratificadas por 30 dias; T4, sementes escarificadas e estratificadas por 30 dias; T5, sementes em temperatura ambiente por 60 dias; T6, sementes em temperatura ambiente por 60 dias e escarificadas após esse período; T7, sementes estratificadas por 60 dias; T8, sementes escarificadas e estratificadas por 60 dias; T9, sementes em temperatura ambiente por 90 dias; T10, sementes em temperatura ambiente por 90 dias e escarificadas após esse período; T11, sementes estratificadas por 90 dias; T12, sementes escarificadas e estratificadas por 90 dias. 
Tabela 2 - Tamanho de amostra (número de plantas) para estimação da média dos caracteres altura de planta, diâmetro do coleto e relação altura de planta/diâmetro do coleto de mudas de nogueira-pecã (Carya illinoinensis), avaliados em 12 tratamentos (TRAT) de superação de dormência de sementes, para as semiamplitudes do intervalo de confiança de 95\% (erros de estimação), iguais a 1\%, $2 \%, \ldots, 15 \%$ da estimativa da média.

\begin{tabular}{|c|c|c|c|c|c|c|c|c|c|c|c|c|c|c|c|}
\hline TRAT $^{(1)}$ & $1 \%$ & $2 \%$ & $3 \%$ & $4 \%$ & $5 \%$ & $6 \%$ & $7 \%$ & $8 \%$ & $9 \%$ & $10 \%$ & $11 \%$ & $12 \%$ & $13 \%$ & $14 \%$ & $15 \%$ \\
\hline & \multicolumn{15}{|c|}{ - } \\
\hline $\mathrm{T} 1$ & 4.315 & 1.079 & 480 & 270 & 173 & 120 & 89 & 68 & 54 & 44 & 36 & 30 & 26 & 23 & 20 \\
\hline $\mathrm{T} 2$ & 1.748 & 437 & 195 & 110 & 70 & 49 & 36 & 28 & 22 & 18 & 15 & 13 & 11 & 9 & 8 \\
\hline $\mathrm{T} 3$ & 6.295 & 1.574 & 700 & 394 & 252 & 175 & 129 & 99 & 78 & 63 & 53 & 44 & 38 & 33 & 28 \\
\hline $\mathrm{T} 4$ & 2.178 & 545 & 242 & 137 & 88 & 61 & 45 & 35 & 27 & 22 & 18 & 16 & 13 & 12 & 10 \\
\hline T5 & 1.973 & 494 & 220 & 124 & 79 & 55 & 41 & 31 & 25 & 20 & 17 & 14 & 12 & 11 & 9 \\
\hline T6 & 2.424 & 606 & 270 & 152 & 97 & 68 & 50 & 38 & 30 & 25 & 21 & 17 & 15 & 13 & 11 \\
\hline $\mathrm{T} 7$ & 2.496 & 624 & 278 & 156 & 100 & 70 & 51 & 39 & 31 & 25 & 21 & 18 & 15 & 13 & 12 \\
\hline $\mathrm{T} 8$ & 1.814 & 454 & 202 & 114 & 73 & 51 & 38 & 29 & 23 & 19 & 15 & 13 & 11 & 10 & 9 \\
\hline T9 & 3.006 & 752 & 334 & 188 & 121 & 84 & 62 & 47 & 38 & 31 & 25 & 21 & 18 & 16 & 14 \\
\hline T10 & 5.772 & 1.443 & 642 & 361 & 231 & 161 & 118 & 91 & 72 & 58 & 48 & 41 & 35 & 30 & 26 \\
\hline T11 & 1.954 & 489 & 218 & 123 & 79 & 55 & 40 & 31 & 25 & 20 & 17 & 14 & 12 & 10 & 9 \\
\hline $\mathrm{T} 12$ & 2.820 & 705 & 314 & 177 & 113 & 79 & 58 & 45 & 35 & 29 & 24 & 20 & 17 & 15 & 13 \\
\hline $\mathrm{T} 1$ & 4.934 & 1.234 & 549 & 309 & 198 & 138 & $\begin{array}{c}\text { iâme } \\
101\end{array}$ & $\begin{array}{c}78 \\
78\end{array}$ & $\begin{array}{l}\text { eto (r } \\
61\end{array}$ & 50 & 41 & 35 & 30 & 26 & 22 \\
\hline $\mathrm{T} 2$ & 2.585 & 647 & 288 & 162 & 104 & 72 & 53 & 41 & 32 & 26 & 22 & 18 & 16 & 14 & 12 \\
\hline $\mathrm{T} 3$ & 2.895 & 724 & 322 & 181 & 116 & 81 & 60 & 46 & 36 & 29 & 24 & 21 & 18 & 15 & 13 \\
\hline $\mathrm{T} 4$ & 2.547 & 637 & 283 & 160 & 102 & 71 & 52 & 40 & 32 & 26 & 22 & 18 & 16 & 13 & 12 \\
\hline T5 & 2.376 & 594 & 264 & 149 & 96 & 66 & 49 & 38 & 30 & 24 & 20 & 17 & 15 & 13 & 11 \\
\hline T6 & 1.789 & 448 & 199 & 112 & 72 & 50 & 37 & 28 & 23 & 18 & 15 & 13 & 11 & 10 & 8 \\
\hline $\mathrm{T} 7$ & 1.825 & 457 & 203 & 115 & 73 & 51 & 38 & 29 & 23 & 19 & 16 & 13 & 11 & 10 & 9 \\
\hline $\mathrm{T} 8$ & 2.538 & 635 & 282 & 159 & 102 & 71 & 52 & 40 & 32 & 26 & 21 & 18 & 16 & 13 & 12 \\
\hline T9 & 3.642 & 911 & 405 & 228 & 146 & 102 & 75 & 57 & 45 & 37 & 31 & 26 & 22 & 19 & 17 \\
\hline $\mathrm{T} 10$ & 5.720 & 1.430 & 636 & 358 & 229 & 159 & 117 & 90 & 71 & 58 & 48 & 40 & 34 & 30 & 26 \\
\hline $\mathrm{T} 11$ & 2.122 & 531 & 236 & 133 & 85 & 59 & 44 & 34 & 27 & 22 & 18 & 15 & 13 & 11 & 10 \\
\hline \multirow[t]{2}{*}{$\mathrm{T} 12$} & 1.183 & 296 & 132 & 74 & 48 & 33 & 25 & 19 & 15 & 12 & 10 & 9 & 7 & 7 & 6 \\
\hline & \multicolumn{15}{|c|}{ - } \\
\hline $\mathrm{T} 1$ & 4.902 & 1.226 & 545 & 307 & 197 & 137 & 101 & 77 & 61 & 50 & 41 & 35 & 30 & 26 & 22 \\
\hline $\mathrm{T} 2$ & 2.476 & 619 & 276 & 155 & 100 & 69 & 51 & 39 & 31 & 25 & 21 & 18 & 15 & 13 & 12 \\
\hline $\mathrm{T} 3$ & 4.623 & 1.156 & 514 & 289 & 185 & 129 & 95 & 73 & 58 & 47 & 39 & 33 & 28 & 24 & 21 \\
\hline $\mathrm{T} 4$ & 987 & 247 & 110 & 62 & 40 & 28 & 21 & 16 & 13 & 10 & 9 & 7 & 6 & 6 & 5 \\
\hline T5 & 2.002 & 501 & 223 & 126 & 81 & 56 & 41 & 32 & 25 & 21 & 17 & 14 & 12 & 11 & 9 \\
\hline T6 & 2.335 & 584 & 260 & 146 & 94 & 65 & 48 & 37 & 29 & 24 & 20 & 17 & 14 & 12 & 11 \\
\hline $\mathrm{T} 7$ & 1.701 & 426 & 189 & 107 & 69 & 48 & 35 & 27 & 21 & 18 & 15 & 12 & 11 & 9 & 8 \\
\hline $\mathrm{T} 8$ & 2.025 & 507 & 225 & 127 & 81 & 57 & 42 & 32 & 25 & 21 & 17 & 15 & 12 & 11 & 9 \\
\hline T9 & 2.400 & 600 & 267 & 150 & 96 & 67 & 49 & 38 & 30 & 24 & 20 & 17 & 15 & 13 & 11 \\
\hline T10 & 2.211 & 553 & 246 & 139 & 89 & 62 & 46 & 35 & 28 & 23 & 19 & 16 & 14 & 12 & 10 \\
\hline T11 & 1.864 & 466 & 208 & 117 & 75 & 52 & 39 & 30 & 24 & 19 & 16 & 13 & 12 & 10 & 9 \\
\hline T12 & 1.521 & 381 & 169 & 96 & 61 & 43 & 32 & 24 & 19 & 16 & 13 & 11 & 9 & 8 & 7 \\
\hline
\end{tabular}

${ }^{(1)} \mathrm{T} 1$, sementes em temperatura ambiente por 30 dias; T2, sementes em temperatura ambiente por 30 dias e escarificadas após esse período; T3, sementes estratificadas por 30 dias; T4, sementes escarificadas e estratificadas por 30 dias; T5, sementes em temperatura ambiente por 60 dias; T6, sementes em temperatura ambiente por 60 dias e escarificadas após esse período; T7, sementes estratificadas por 60 dias; T8, sementes escarificadas e estratificadas por 60 dias; T9, sementes em temperatura ambiente por 90 dias; T10, sementes em temperatura ambiente por 90 dias e escarificadas após esse período; T11, sementes estratificadas por 90 dias; T12, sementes escarificadas e estratificadas por 90 dias. 


\section{REFERENCIAS}

BUSSAB, W.O.; MORETTIN, P.A. Estatística básica. 7.ed. São Paulo: Saraiva, 2011. 540p.

CARGNELUTTI FILHO, A. et al. Dimensionamento amostral para avaliação de altura e diâmetro de mudas de Cabralea Canjerana. Ciência Rural, v.42, p.1204-1211, 2012. Disponível em: <http:// www.scielo.br/pdf/cr/v42n7/a19912cr5829.pdf>. Acesso em: 19 mar. 2014. doi: 10.1590/S0103-84782012000700011.

CRUZ, C.D. GENES - a software package for analysis in experimental statistics and quantitative genetics. Acta
Scientiarum Agronomy, v.35, p.271-276, 2013. Disponível em: <http://periodicos.uem.br/ojs/index.php/ActaSciAgron/article/ view/21251/pdf>. Acesso em: 19 mar. 2014.

SILVEIRA, B.D. et al. Aleatoriedade e tamanho da amostra em mudas de Pinus taeda L. Ceres, v.56, p.730-735, 2009. Disponível em: 〈http://www.ceres.ufv.br/ceres/revistas/V56N006P05108.pdf>. Acesso em: 19 mar. 2014.

ZANON, M.L.B. et al. Tamanho de amostra para experimentos de Eucalyptus saligna SMITH em viveiro. Ciência Florestal, v.7, p.133138, 1997. Disponível em: <http://coral.ufsm.br/cienciaflorestal/ artigos/v7n1/art11v7n1.pdf $>$. Acesso em: 19 mar. 2014.

Ciência Rural, v.44, n.12, dez, 2014. 